\title{
BMJ Open Comprehensive mapping of NICU developmental care nursing interventions and related sensitive outcome indicators: a scoping review protocol
}

\author{
Marjolaine Héon (1) , ${ }^{1,2}$ Marilyn Aita (1) ,1,2,3 Andréane Lavallée (1) ,1,3 \\ Gwenaëlle De Clifford-Faugère (D) ,1,3 Geneviève Laporte (D) ,, ${ }^{1,3}$ Annie Boisvert, ${ }^{1,4}$ \\ Nancy Feeley (D) 2,5,6
}

To cite: Héon $\mathrm{M}$,

Aita M, Lavallée A, et al. Comprehensive mapping of NICU developmental care nursing interventions and related sensitive outcome indicators: a scoping review protocol. BMJ Open 2022;12:e046807. doi:10.1136/ bmjopen-2020-046807

- Prepublication history and additional supplemental material for this paper are available online. To view these files, please visit the journal online (http://dx.doi.org/10.1136/ bmjopen-2020-046807).

Received 09 November 2020 Accepted 11 November 2021

Check for updates

(C) Author(s) (or their employer(s)) 2022. Re-use permitted under CC BY-NC. No commercial re-use. See rights and permissions. Published by BMJ.

For numbered affiliations see end of article.

Correspondence to Dr Marjolaine Héon; marjolaine.heon@umontreal. ca and

Dr Marjolaine Héon; marjolaine.heon@umontreal.ca

\section{ABSTRACT}

Introduction Neurodevelopmental outcomes of preterm infant are still a contemporary concern. To counter the detrimental effects resulting from the hospitalisation in the neonatal intensive care unit (NICU), developmental care (DC) interventions have emerged as a philosophy of care aimed at protecting and enhancing preterm infant's development and promoting parental outcomes. In the past two decades, many authors have suggested DC models, core measures, practice guidelines and standards of care but outlined different groupings of interventions rather than specific interventions that can be used in NICU clinical practice. Moreover, as these DC interventions are mostly implemented by neonatal nurses, it would be strategic and valuable to identify specific outcome indicators to make visible the contribution of NICU nurses to DC.

Objectives The overarching objective of this review is to identify the nature, range, and extent of the literature regarding DC nursing interventions for preterm infants in the NICU. The secondary twofold objectives are to highlight interventions that fall into identified categories of DC interventions and suggest nursing-sensitive outcome indicators related to DC interventions in the NICU.

Inclusion criteria Papers reporting on or discussing a DC nursing intervention during NICU hospitalisation will be included.

Methods and analysis The Joanna Briggs Institute's methodology for scoping reviews will be followed. CINAHL, MEDLINE, Embase, PubMed, Web of Science, Scopus, ProQuest and Psyclnfo databases from 2009 to the present will be searched. Any type of paper, published in English or French, will be considered. Study selection and data extraction will be conducted by pairs of two review authors independently. A qualitative content analysis will be conducted. Ethics and dissemination No Institutional Review Board ethical approbation is needed. Results of this review will be presented in scientific meetings and published in refereed papers.

\section{Strengths and limitations of this study}

First scoping review that aims to identify nursingsensitive outcome indicators related to developmental care interventions in the neonatal intensive care unit (NICU).

- New comprehensive and inclusive categories of developmental care interventions orient this scoping review.

- This scoping review protocol follows the recommendations of the Joanna Briggs Institute for the conduct of systematic and rigorous reviews.

- As the scope of this review is large and the literature search strategy is very sensitive, it will include all relevant literature but might lack specificity.

- The scope of this review is limited to developmental care interventions delivered by neonatal nurses in the NICU.

\section{INTRODUCTION}

Short and long-term neurodevelopmental outcomes are still a contemporary concern for infants who are born preterm, that is, before the 37 th week of gestation (WG) is completed. ${ }^{1-3}$ Compared with term infants, school-age children born preterm have significant deficits in mathematics and reading, ${ }^{1}$ present with lower IQs $^{4}$ and are at increased risk for anxiety and hyperactivity disorders. ${ }^{5}$ Throughout adulthood, young adults born preterm are still facing significant social-emotional difficulties, ${ }^{3}$ present with lower $\mathrm{IQ}^{6}$ are even at increased risk for autistic symptoms ${ }^{7}$ and obtain lower scores on neuropsychological tests. ${ }^{2}$ The hospitalisation in the neonatal intensive care unit (NICU) has been identified as a strategic period to implement interventions to protect and optimise preterm infant's neurodevelopment. 8 In fact, the most important maturation processes of the 
central nervous system occur between the 24th and the 40th WG. $^{9}$

The concept of developmental care (DC), based on the work of Dr Heidelise Als, ${ }^{10}$ is a neuroprotective NICU care philosophy that ultimately intends to promote optimal health outcomes in preterm infants and their families. ${ }^{11-13}$ An important aspect of DC is the individualisation of care that should match each infant and their family's needs, ${ }^{13} 14$ with the aim of lowering parental stress and maximising their adaptation, as well as improving the infant's development. ${ }^{14}$ DC has proven to be effective in promoting mental and psychomotor development, ${ }^{15}$ neurobehaviour and oral feeding ${ }^{16}$; reducing NICU length of stay ${ }^{16}$; and lowering parental stress and anxiety. ${ }^{17}$ Thus, it appears important to scope the DC literature to identify specific interventions neonatal nurses can implement daily in their practice in order to optimise preterm infants and families' outcomes.

However, it is unclear which specific interventions DC encompasses. Different authors have suggested groupings of DC interventions, which have been referred to as a DC model,${ }^{18}{ }^{19}$ practice guidelines, ${ }^{12} 20$ conceptual model, ${ }^{21}$ core measures ${ }^{22}$ and standards of care. ${ }^{23-34}$ For example, in their DC model, Altimier and Phillips ${ }^{18} 19$ suggest there are seven neuroprotective DC core measures including: the healing environment (physical, sensory, smell/test, sound/noise, light), partnering with families, positioning and handling the infant, safeguarding sleep, minimising stress and pain, protecting skin and optimising nutrition. The National Association of Neonatal Nurses' practice guidelines ${ }^{12}$ and Coughlin $e t a t^{2}$ both suggest five core measures, such as: protected sleep, assessment and management of stress and pain, developmentally supportive activities of daily living, family-centred care and creating a healing environment. Gibbins $e t a l^{21}$ in their universe of DC conceptual model, suggest that DC interventions fit into 12 categories: monitoring/assessing, feeding, positioning, infection control, safety, comfort, thermoregulation, skin care, respiratory care, family, staff and environment. Lindacher et a $t^{23}$ provide 96 European standards of care for newborn health on 11 overarching topic areas, including 10 standards on infantcentred and family-centred $\mathrm{DC}^{24}$ : case management and transition to home ${ }^{25}$; clinical consultation and supervision for healthcare professionals on supporting families ${ }^{26}$; education and training for infant-centred and family-centred $\mathrm{DC}^{27}$; family access ${ }^{28}$; family support services ${ }^{29}$; management of the acoustic environment ${ }^{30}$; parental involvement ${ }^{31}$; support for parental-infant bonding ${ }^{32}$; supportive sensory environment ${ }^{33}$; and very early and continuous skin-to-skin contact. ${ }^{34}$ Finally, Browne et $a l^{35}$ identify six key practice domains of infant and family-centred DC in the intensive care unit: systems thinking; positioning and touch for the newborn; sleep and arousal interventions for the newborn; skin-to-skin contact with intimate family members; reducing and managing pain and stress in newborns and families; and management of feeding, eating and nutrition delivery. Still, it remains ambiguous which specific interventions fit into those global categories. For example, Jebreili et $a l^{36}$ evaluated the effectiveness of an olfactive stimulation intervention to manage procedural pain of preterm infants in the NICU. Although this intervention aims at reducing the preterm infant's pain, the authors do not identify it as a DC intervention, nor does it appear in any of the aforementioned groupings. To develop categories of DC interventions that are comprehensive for nursing, and inclusive, we propose that DC interventions fall into eight categories. Our classification encompasses all categories suggested by the various above-mentioned authors: family-centred care, feeding, positioning and handling, reduction and management of pain, sensory control, sensory stimulation, skin and routine care and sleep protection.

Even though DC is a multidisciplinary approach, ${ }^{13}$ DC interventions are primarily delivered by neonatal nurses. ${ }^{14}$ Indeed, by virtue of their field of practice, their professional skills and their unique proximity in the healthcare experience of preterm infants and their families, neonatal nurses are strategically positioned to implement DC interventions in the NICU. ${ }^{1437}$ However, the invisibility of the contribution of neonatal nursing to infants' health is a major concern, because it does not allow to distinguish their distinctive and exclusive role in NICU clinical practice, ${ }^{38}$ along with how their involvement may favourably influence infants' health outcomes. The visibility of their specific contribution is compromised by the absence of a global portrait of nursing-sensitive outcome indicators that would allow us to better understand the effects and benefits of DC interventions in the NICU. Introduced by Maas $e t a l^{39}$ the concept of 'nursing-sensitive outcome indicator' refers to the distinct and measurable change in patient's state, behaviour or perception as a result of a nursing intervention. In the past years, several initiatives to identify nursing-sensitive outcome indicators have emerged. ${ }^{40-43}$ Although these authors have identified nursing-sensitive outcome indicators, the former remain generic and some of these indicators, such as falls and incontinence, are not transferable to a neonatal population. Consequently, the range of implemented DC interventions remains unknown and the absence of specific nursingsensitive outcome indicators related to these neonatal DC interventions prevents the evaluation of nursing contribution to preterm infants and families' well-being. A database of outcome indicators for neonatology was developed by The Canadian Neonatal Network. ${ }^{44}$ Nonetheless, the authors of this report did not specifically take into account outcome indicators that are specific to neonatal nursing DC interventions but rather observed medical outcomes such as sepsis, survival rates and cardiovascular complication rates. Moreover, 11 nursing-sensitive quality indicators for the NICU were developed in a study by Chen $e t a l l^{45}$ but then again, these indicators are general to NICU care (ie, rate of compliance to proper hand washing, rate of nosocomial infections, etc) and not specifically related to DC nursing interventions. Thus, there is a pressing need for a comprehensive mapping of nursing-sensitive outcome indicators with regard to DC interventions. Such effort is essential to identify outcome indicators that have been reported so far in the scientific literature and those that require further assessment, as well as to circumscribe the effects of DC interventions delivered 
by nurses on preterm infants and families' health and development.

A preliminary search of PROSPERO, MEDLINE, the Cochrane Database of Systematic Reviews and the Joanna Briggs Institute Database of Systematic Reviews and Implementation Reports was conducted. Some systematic reviews have looked at the effectiveness of specific DC programmes, such as the Newborn Individualized Developmental Care and Assessment Program, ${ }^{15}$ or the effectiveness of interventions on preterm infant's development or health outcomes. ${ }^{1646-48}$ To our knowledge, no current reviews scoping the literature on DC nursing interventions have been published or are underway.

Based on this lack of evidence, the primary objective for this scoping review is to: (1) identify the nature, range, and extent of the literature regarding DC nursing interventions in the NICU. The secondary objectives are twofold: (2) to highlight DC interventions that fall into our eight identified categories of DC nursing interventions; and (3) suggest nursing-sensitive outcome indicators related to DC interventions.

\section{REVIEW QUESTIONS}

The primary question guiding this scoping review is the following: What is the nature, range, and the extent of the literature regarding DC nursing interventions for preterm infants and families in the NICU?

The secondary questions addressed in this scoping review are: What are the interventions that have been associated with our eight categories of DC nursing interventions in the NICU? What are the nursing-sensitive outcome indicators related to DC interventions in the NICU?

\section{METHODS}

The proposed scoping review will be conducted in accordance with the Joanna Briggs Institute (JBI) methodology for scoping reviews. ${ }^{49}$ Moreover, as suggested by the JBI, this protocol is based on the Preferred Reporting Items for Systematic Reviews and MetaAnalyses extension for Scoping Reviews Checklist (see online supplemental file 1). ${ }^{50}$

\section{Inclusion criteria}

Participants

We will consider studies that included preterm infants or their parents. For this review, we will consider the definition suggested by the WHO stating that preterm infants are infants born before the 37 th WG is completed. ${ }^{51}$

\section{Concept}

We will consider studies that relate to one or many nursing interventions and that fit in one or more of our identified categories of DC. For this review, as stated before, we established eight categories of DC nursing interventions that encompass all major categories that are suggested in other DC model, ${ }^{18}{ }^{19}$ practice guidelines, ${ }^{12}{ }^{20}$ conceptual model, ${ }^{21}$ core measures ${ }^{22}$ and standards of care. ${ }^{23-34}$ Those eight categories are (see table 1): sensory control, sensory stimulation, familycentred care, positioning and handling, sleep protection, comfort, skin and routine care, and feeding. In other words, any paper that discusses or is related to one or more specific nursing interventions that fall into one of these eight categories will be included in this scoping review. Also, a DC intervention will be qualified as a nursing intervention if it is delivered by nurses or if the intervention could be delivered by nurses as per their field of practice. We will also consider interventions if they are delivered by parents themselves. For example, a study evaluating a massage intervention (sensory stimulation) delivered by nurses would be eligible for inclusion in this review. On the other hand, a study evaluating the effects of specific macronutrients or micronutrients (feeding) would be excluded because it would be qualified as a medical intervention.

As for outcomes, all outcomes measured during the NICU hospitalisation will be considered for the inclusion of papers in the review. Papers that do not report any outcome as well as conference abstracts will be excluded.

\section{Context}

We will consider papers that discuss DC nursing interventions for preterm infants and their families during the NICU hospitalisation only, that being before the infant is discharged home or transferred to another care unit.

\section{Types of sources}

In this scoping review, we will consider quantitative, qualitative and mixed methods study designs for inclusion. In addition, literature reviews, text and opinion papers, practice guidelines and theoretical papers will be considered. Articles published in French or English will be included. Articles published from CINAHL, MEDLINE, Embase, PubMed, Web of Science, Scopus, ProQuest and PsycInfo databases from 2009 to the present will be included as modern DC interventions have mostly evolved over the last decade.

\section{Search strategy}

The search strategy will aim to locate both published and unpublished primary studies, reviews and opinion papers pertaining to DC nursing interventions in the NICU. An initial search strategy was developed and piloted with a librarian, based onMedical Subject Headings (MeSH) databases. A full search strategy for CINAHL, MEDLINE, Embase, PubMed, Web of Science, Scopus, ProQuest and PsycInfo (see online supplemental file 2, table S1) was then proposed. Key concepts include neonatology, DC and nursing. The 


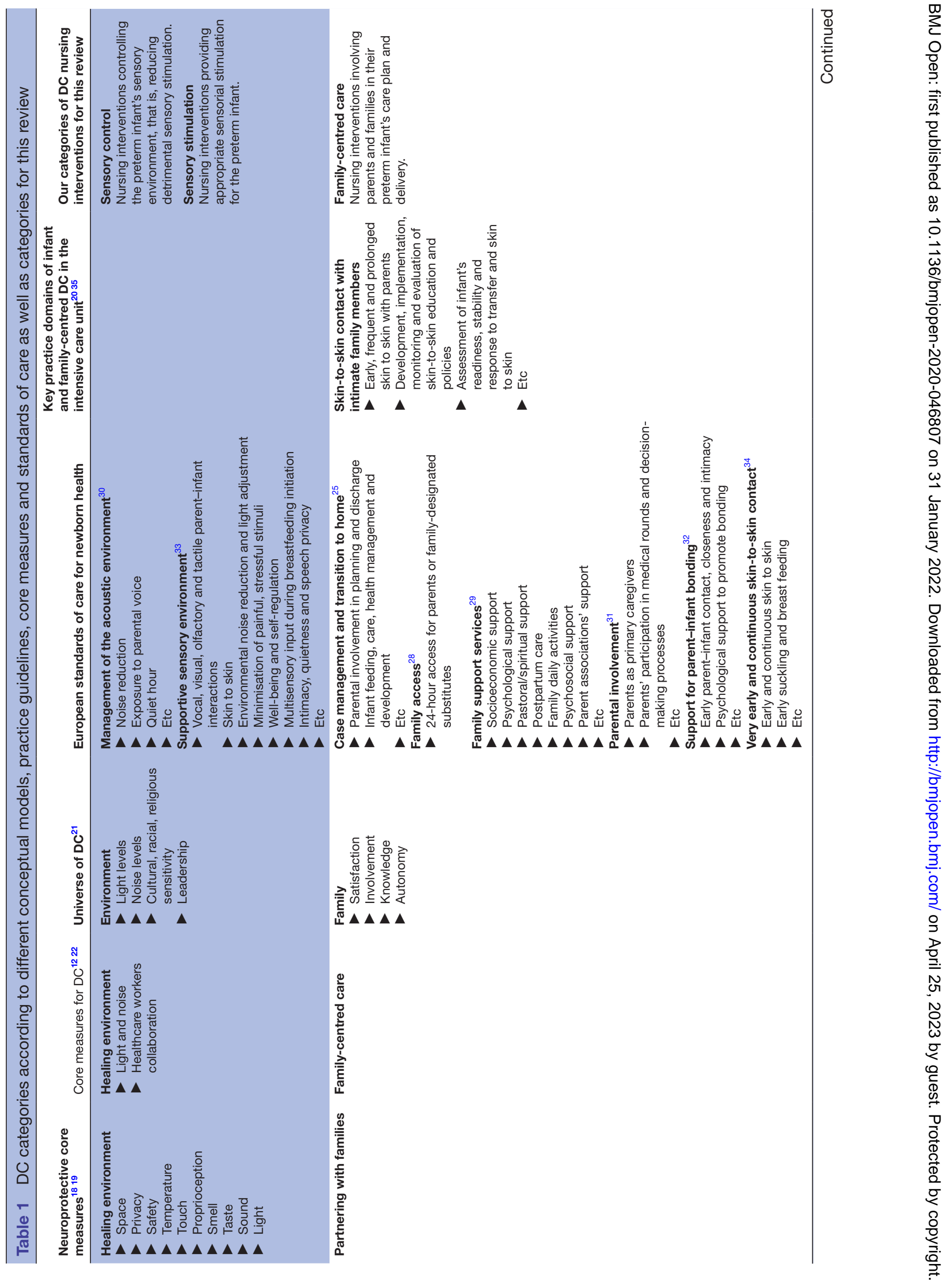




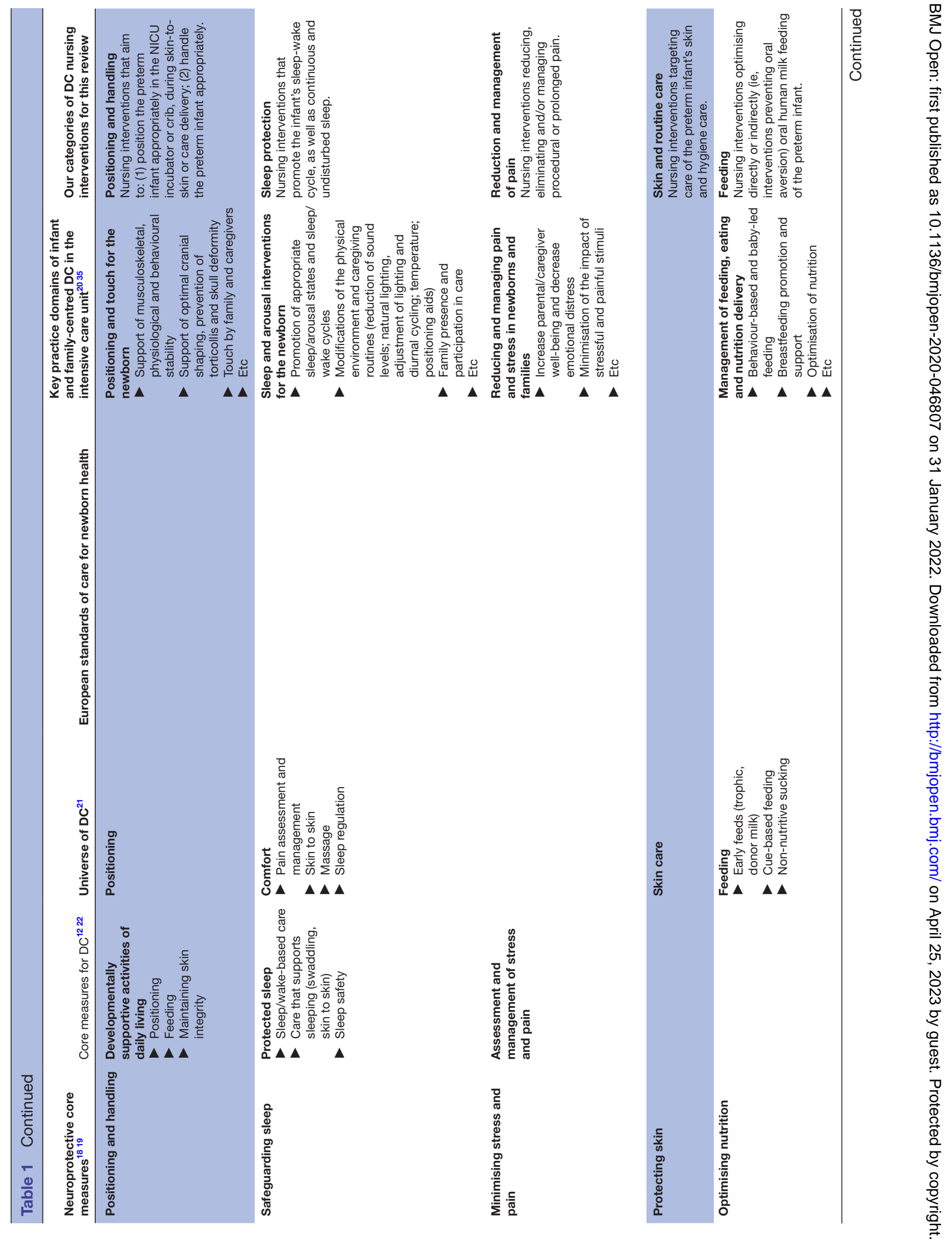




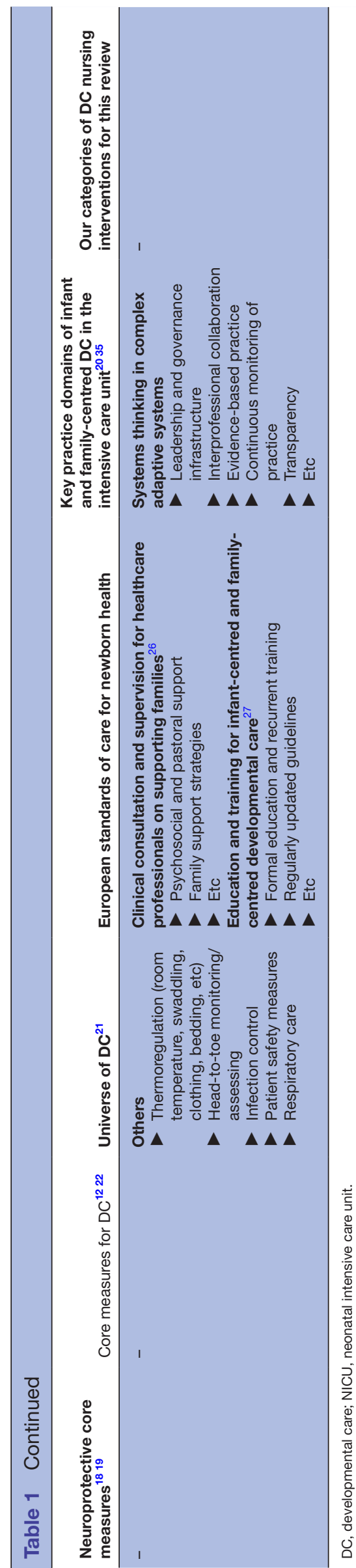

search strategy will be restricted to the last 12 years (2009-2021) due to the rise in publication in DC in the recent years. The search strategy, including all identified keywords and index terms, will be adapted for each included information source.

\section{Information sources}

In addition to the proposed databases (CINAHL, MEDLINE, Embase, PubMed, Web of Science, Scopus, ProQuest and PsycInfo), several grey literature sources will be hand searched, including Google Scholar, the Grey Guide and clinical trial registries (ClinicalTrials.gov, clin icaltrialregister.eu, isrctn.com, anzctr.org.au). Reference lists will also be analysed when appropriate to identify additional papers. Finally, a monthly bibliographic watch on DC prepared by our centre's librarian (https://soinsdudeveloppement.wordpress.com/) will be reviewed and analysed for potential papers. Furthermore, authors with incomplete records will be contacted as needed to obtain supplemental information.

\section{Study selection}

Following the search, all identified records will be collated and uploaded into Covidence systematic review software V.1528 (Veritas Health Innovation, Melbourne, Australia; www.covidence.org) and duplicates removed. Seven review authors (MH, MA, AL, GDC-F, GL, AB, NF), in teams of two, will then screen titles and abstracts against the inclusion criteria as a means to pilot the specificity of inclusion criteria. Each reviewer will screen 250 articles to assess the criteria's performance and the team will further refine them accordingly. Criteria will be piloted again until performance is deemed adequate by all reviewers. Initial screening will be completed by one independent reviewer. Potentially relevant papers will be retrieved in full and their citation details imported into Covidence. The full text of selected citations will be assessed in detail against the inclusion criteria by the same seven review authors, in teams of two independently. Similar to the initial screening, inclusion criteria will be piloted and further refined before completing the full-text selection process. Reasons for exclusion of full-text papers that do not meet the inclusion criteria will be recorded and reported in the scoping review. Any disagreements that arise between the reviewers at each stage of the selection process will be resolved with a third review author. The results of the search will be reported in full in the final scoping review and presented in a Preferred Reporting Items for Systematic Reviews and Meta-Analyses flow diagram. ${ }^{52}$

\section{Data extraction}

Data will be extracted from papers included in the scoping review by two independent reviewers using a data extraction tool developed by the review authors. Any disagreements that arise between the reviewers will be resolved through discussion, or with a third reviewer. The draft data extraction tool will be modified and revised as 
necessary during the pilot phase of extracting data from the first 20 included papers. Authors of papers will be contacted to request missing or additional data, where required. Modifications, if needed, will be detailed in the full scoping review.

\section{Data items}

To answer to our first two review questions, those being to describe the nature, range, and extent of the literature as well as specific DC nursing interventions, the data extracted will include:

1. Descriptive data: authors, year of publication, country of origin, type of article and aim.

2. Methodological data: study design (if applicable), population (eg, gestational age of the preterm infants at birth, inclusion or not of parents).

3. Data about the specific DC nursing intervention: category of DC intervention to which it pertains according to our proposed classification, details about the intervention as per the Description and Replication checklist, ${ }^{53}$ when applicable-the intervention, the materials, the procedures, the provider(s), the modes of delivery, where, when and how much (frequency, duration and dose) as well as possibilities for tailoring the intervention.

A second data extraction process will be conducted to answer to our third review question, that is, to highlight nursing-sensitive outcome indicators related to DC interventions. Thus, in order to identify nursingsensitive outcome indicators, we will extract the following data only for papers with an experimental design:

4. Outcome data: outcomes measured, timing of outcome measure and reported results.

\section{Critical appraisal and secondary data synthesis}

Critical appraisal of included papers is not mandatory according to the scoping review JBI methodology. ${ }^{49}$ Nevertheless, as per our third review question, we will critically appraise all studies with an experimental design using the JBI Checklist for Randomized Controlled Trials. ${ }^{54}$ Two independent review authors will complete the checklist for each experimental study and disagreements will be resolved by a third review author.

A secondary qualitative data synthesis of the outcomes reported in experimental studies will be conducted in order to highlight nursing-sensitive outcome indicators related to DC interventions in the NICU. Provisional outcome indicators will be shared with experts with a clinical or academic background in the field of quality of care and neonatology so they can provide guidance and, ultimately, validation. Detailed methodology that pertains to the third objective will be reported in the results paper.

\section{Data presentation}

For our primary and first secondary objectives, the extracted data will be presented in tabular form. A narrative summary will accompany the tabulated results and will describe how the results relate to the reviews' objectives and questions. As per our other secondary objective, data will be presented narratively.

\section{Patient and public involvement}

Patients and members of the public were not involved in the development of this protocol.

\section{ETHICS AND DISSEMINATION}

As this is a literature review project using already collected and published data, it will not be necessary to seek ethical approval from an Institutional Review Board. Results of this scoping review will be presented in scientific meetings and published in refereed papers. Our three objectives will be reported in three results papers.

\section{Author affiliations}

${ }^{1}$ Faculty of Nursing, Université de Montréal, Montreal, Quebec, Canada

${ }^{2}$ Quebec Network on Nursing Intervention Research/Réseau de Recherche en Interventions en Sciences Infirmières du Québec (RRISIQ), Montreal, Quebec, Canada

${ }^{3}$ Research Centre, CHU Sainte-Justine, Montreal, Quebec, Canada

${ }^{4} \mathrm{NICU}, \mathrm{CHU}$ Sainte-Justine, Montreal, Quebec, Canada

${ }^{5}$ Ingram School of Nursing, McGill University, Montreal, Quebec, Canada

${ }^{6}$ Centre for Nursing Research and Lady Davis Institute for Medical Research, Jewish General Hospital, Montreal, Quebec, Canada

\section{Twitter Nancy Feeley @NIfeeleyN}

Acknowledgements We would like to acknowledge the contribution of Valérie Lebel and Assia Mourid for the search strategy.

Contributors $\mathrm{MH}(\mathrm{PI})$ drafted the first version of the review protocol revised by MA and NF (Co-Is) to obtain funding from RRISIQ. AL wrote the first version of this manuscript with the contribution of MH, MA, GL, GDC-F and NF. All authors read and approved the final version. $\mathrm{MH}, \mathrm{GL}$ and $\mathrm{AB}$ drafted the initial search strategy.

Funding This scoping review is funded by the Quebec Network on Nursing Intervention Research/Réseau de Recherche en Interventions en Sciences Infirmières du Québec (RRISIQ).

Disclaimer The funders are not involved in any form in the review process. Competing interests None declared.

Patient consent for publication Not required.

Provenance and peer review Not commissioned; externally peer reviewed.

Supplemental material This content has been supplied by the author(s). It has not been vetted by BMJ Publishing Group Limited (BMJ) and may not have been peer-reviewed. Any opinions or recommendations discussed are solely those of the author(s) and are not endorsed by BMJ. BMJ disclaims all liability and responsibility arising from any reliance placed on the content. Where the content includes any translated material, BMJ does not warrant the accuracy and reliability of the translations (including but not limited to local regulations, clinical guidelines, terminology, drug names and drug dosages), and is not responsible for any error and/or omissions arising from translation and adaptation or otherwise.

Open access This is an open access article distributed in accordance with the Creative Commons Attribution Non Commercial (CC BY-NC 4.0) license, which permits others to distribute, remix, adapt, build upon this work non-commercially, and license their derivative works on different terms, provided the original work is properly cited, appropriate credit is given, any changes made indicated, and the use is non-commercial. See: http://creativecommons.org/licenses/by-nc/4.0/.

\section{ORCID iDs}

Marjolaine Héon http://orcid.org/0000-0001-7450-1797

Marilyn Aita http://orcid.org/0000-0002-6197-8796

Andréane Lavallée http://orcid.org/0000-0001-5702-3084

Gwenaëlle De Clifford-Faugère http://orcid.org/0000-0002-9719-5531

Geneviève Laporte http://orcid.org/0000-0002-3612-8546

Nancy Feeley http://orcid.org/0000-0003-2836-4116 


\section{REFERENCES}

1 McBryde M, Fitzallen GC, Liley HG, et al. Academic outcomes of school-aged children born preterm: a systematic review and metaanalysis. JAMA Netw Open 2020;3:e202027.

2 O'Reilly H, Johnson S, Ni Y, et al. Neuropsychological outcomes at 19 years of age following extremely preterm birth. Pediatrics 2020;145:e20192087.

3 Pyhälä R, Wolford E, Kautiainen $\mathrm{H}$, et al. Self-Reported mental health problems among adults born preterm: a meta-analysis. Pediatrics 2017;139:e20162690.

4 Wolke D, Strauss VY-C, Johnson S, et al. Universal gestational age effects on cognitive and basic mathematic processing: 2 cohorts in 2 countries. J Pediatr 2015;166:1410-6.

5 Treyvaud K, Ure A, Doyle LW, et al. Psychiatric outcomes at age seven for very preterm children: rates and predictors. J Child Psychol Psychiatry 2013;54:772-9.

6 Breeman LD, Jaekel J, Baumann N, et al. Preterm cognitive function into adulthood. Pediatrics 2015;136:415-23.

7 Eryigit-Madzwamuse S, Strauss V, Baumann N, et al. Personality of adults who were born very preterm. Arch Dis Child Fetal Neonatal Ed 2015;100:F524-9.

8 Lavallée A, De Clifford-Faugère G, Garcia C, et al. Part 1: narrative overview of developmental care interventions for the preterm newborn. Journal of Neonatal Nursing 2019;25:3-8.

9 Volpe JJ. Brain injury in premature infants: a complex Amalgam of destructive and developmental disturbances. Lancet Neurol 2009;8:110-24.

10 Als $\mathrm{H}$. Toward a synactive theory of development: promise for the assessment and support of infant individuality. Infant Ment Health $\mathrm{J}$ 1982;3:229-43.

11 Als H. Developmental care in the newborn intensive care unit. Curr Opin Pediatr 1998;10:138-42.

12 Kenner C, McGrath JM. Developmental care of newborns and infants. 2 ed. Illinois: National Association of Neonatal Nurses, 2010.

13 Aita M, Snider L. The art of developmental care in the NICU: a concept analysis. J Adv Nurs 2003;41:223-32.

14 Macho P. Individualized developmental care in the NICU: a concept analysis. Adv Neonatal Care 2017;17:162-74.

15 Ohlsson A, Jacobs SE. NIDCAP: a systematic review and meta-analyses of randomized controlled trials. Pediatrics 2013;131:e881-93.

16 Symington A, Pinelli J. Developmental care for promoting development and preventing morbidity in preterm infants. Cochrane Database Syst Rev 2006:CD001814.

17 Ding X, Zhu L, Zhang R, et al. Effects of family-centred care interventions on preterm infants and parents in neonatal intensive care units: a systematic review and meta-analysis of randomised controlled trials. Aust Crit Care 2019;32:63-75.

18 Altimier L, Phillips RM. The neonatal integrative developmental care model: seven neuroprotective core measures for FamilyCentered developmental care. Newborn and Infant Nursing Reviews 2013;13:9-22.

19 Altimier L, Phillips R. The neonatal integrative developmental care model: advanced clinical applications of the seven core measures for neuroprotective Family-centered developmental care. Newborn and Infant Nursing Reviews 2016;16:230-44.

20 University of Notre Dame. Report of the first consensus conference on Standards, competencies and best practices for infant and Family-Centered developmental care in the intensive care unit, 2019. Available: https://nicudesign.nd.edu/nicu-care-standards/

21 Gibbins S, Hoath SB, Coughlin M, et al. The universe of developmental care: a new conceptual model for application in the neonatal intensive care unit. Adv Neonatal Care 2008;8:141-7.

22 Coughlin M, Gibbins S, Hoath S. Core measures for developmentally supportive care in neonatal intensive care units: theory, precedence and practice. J Adv Nurs 2009;65:2239-48.

23 Lindacher V, Altebaeumer P, Marlow N, et al. European standards of care for newborn Health-A project protocol. Acta Paediatr 2021;110:1433-8.

24 European Foundation for the Care of Newborn Infants. Infant- \& family-centered development care, 2018.

25 EFCNI, Damhuis G, König K, et al., European standards of care for newborn health: case management and transition to home, 2018.

26 EFCNI, Ahlqvist-Björkroth S, Westrup B, et al., European standards of care for newborn health: clinical consultation and supervision for healthcare professionals on supporting families, 2018.
27 EFCNI, Warren I, Westrup B, et al., European standards of care for newborn health: education and training for infant- and family-centred developmental care (IFCDC), 2018.

28 EFCNI, Westrup B, Kuhn P, et al., European standards of care for newborn health: family access, 2018.

29 EFCNI, Westrup B, Kuhn P, et al., European standards of care for newborn health: family support services, 2018.

30 EFCNI, Sizun J, Hallberg B, et al., European standards of care for newborn health: management of the acoustic environment, 2018.

31 EFCNI, Pallás-Alonso C, Westrup B, et al., European standards of care for newborn health: parental involvement, 2018.

32 EFCNI, Montirosso R, Westrup B, et al., European standards of care for newborn health: support for parental-infant bonding, 2018.

33 EFCNI, Kuhn P, Westrup B, et al., European standards of care for newborn health: supportive sensory environment, 2018.

34 EFCNI, Bergman NJ, Westrup B, et al., European standards of care for newborn health: very early and continuous skin-to-skin contact, 2018.

35 Browne JV, Jaeger CB, Kenner C, et al. Executive summary: Standards, competencies, and recommended best practices for infant- and family-centered developmental care in the intensive care unit. J Perinatol 2020;40:5-10.

36 Jebreili M, Neshat H, Seyyedrasouli A, et al. Comparison of Breastmilk odor and Vanilla odor on mitigating premature infants response to pain during and after venipuncture. Breastfeed Med 2015;10:362-5.

37 Lavallée A, De Clifford-Faugère G, Garcia C, et al. Part 2: practice and research recommendations for quality developmental care in the NICU. Journal of Neonatal Nursing 2019;25:160-5.

38 Eklund W, Kenner C. Council of international neonatal nurses (COINN) global neonatal provider database initiative (CGNPD): results from an implementation focus group. Adv Neonatal Care 2015;15:407-15.

39 Maas ML, Johnson M, Moorhead S. Classifying nursing-sensitive patient outcomes. Image J Nurs Sch 1996;28:295-302.

40 Dubois CA, D'Amour D, Brault I, et al. Indicateurs prioritaires pour évaluer la contribution infirmière la qualité des soins: revue systématique des écrits. SIDIIEF, 2015.

41 National Quality Forum. National voluntary consensus standards for Nursing-Sensitive care: an initial performance measure set, 2004.

42 Danielis M, Palese A, Terzoni S, et al. What nursing sensitive outcomes have been studied to-date among patients cared for in intensive care units? findings from a scoping review. Int J Nurs Stud 2020;102:103491.

43 Montalvo I. The National database of nursing quality IndicatorsTM (NDNQIR). OJIN 2007;12.

44 The Canadian Neonatal Network,. Annual report, 2019.

45 Chen L, Huang L-H, Xing M-Y, et al. Using the Delphi method to develop nursing-sensitive quality indicators for the NICU. J Clin Nurs 2017;26:502-13.

46 Legendre V, Burtner PA, Martinez KL, et al. The evolving practice of developmental care in the neonatal unit: a systematic review. Phys Occup Ther Pediatr 2011;31:315-38.

47 Burke S. Systematic review of developmental care interventions in the neonatal intensive care unit since 2006. J Child Health Care 2018;22:269-86.

48 Soleimani F, Azari N, Ghiasvand H, et al. Do NICU developmental care improve cognitive and motor outcomes for preterm infants? A systematic review and meta-analysis. BMC Pediatr 2020;20:67.

49 Peters MDJ, Godfrey C, Mclnerney P, et al. Chapter 11: Scoping Reviews. In: Aromataris E, Munn Z, eds. JBI manual for evidence synthesis, JBI, 2020. https://synthesismanual.jbi.global. https://doi. org/10.46658/JBIMES-20-12

50 Tricco AC, Lillie E, Zarin W, et al. PRISMA extension for scoping reviews (PRISMA-ScR): checklist and explanation. Ann Intern Med 2018;169:467-73.

51 World Health Organization. Preterm birth, 2020. Available: https:// www.who.int/news-room/fact-sheets/detail/preterm-birth\#: :text= Preterm\%20is\%20defined\%20as\%20babies,preterm\%20(28\% 20to\%2032\%20weeks)

52 Moher D, Liberati A, Tetzlaff J, et al. Preferred reporting items for systematic reviews and meta-analyses: the PRISMA statement. J Clin Epidemiol 2009;62:1006-12.

53 Hoffmann TC, Glasziou PP, Boutron I, et al. Better reporting of interventions: template for intervention description and replication (TIDieR) checklist and guide. BMJ 2014;348:g1687.

54 Joanna Briggs Institute checklist for randomized controlled trials 2017. 